\title{
AVALIAÇÃO DA EFICÁCIA DE EXTRATOS OLEOSOS DE FRUTOS VERDES E MADUROS DE CINAMOMO (Melia azedarach) SOBRE Rhipicephalus (Boophilus) microplus (ACARI: IXODIDAE)
}

\author{
LORENA A. DIAS DE SOUSA ${ }^{1}$; SARA F. SOARES ${ }^{1}$; HÉLIO B. PIRES JÚNIOR ${ }^{1}$; PEDRO H. FERRI ${ }^{2}$, \\ LÍGIA M. FERREIRA BORGES ${ }^{1}$
}

\begin{abstract}
SOUSA, L.A.D. DE; SOARES, S.F.; PIRES JÚNIOR, H.B.; FERRI, P.H.; BORGES, L.M.F. [Evaluation of efficacy of ripe and unripe fruit oil extracts of Melia azedarach against Rhipicephalus (Boophilus) microplus (Acari: ixodidae)]. Avaliação da eficácia de extratos oleosos de frutos verdes e maduros de cinamomo (Melia azedarach) sobre Rhipicephalus (Boophilus) microplus (Acari: Ixodidae). Revista Brasileira de Parasitologia Veterinária, v. 17, n. 1, p.36-40, 2008. Centro de Parasitologia Veterinária, Escola de Veterinária, Universidade Federal de Goiás (UFG), Campus II, Samambaia, Caixa Postal 131, Goiânia, GO 74001-970, Brasil. E-mail: lorenavet2@gmail.com

Hexanic extracts gotten of the fruits of cinamomo (Melia azedarach) were tested on engourged females and larvae of Rhipicephalus (Boophilus) microplus. Four extracts were produced: one of unripe fruits and three of ripe fruits, being one with the fruits immediately processed, another one with fruits stored for five months and one tested after storage of the extract for 2 (two) years at $4^{\circ} \mathrm{C}$. The tests were made in triplicate, in $0.25 \%-0.0156 \%$ dilutions. The effectiveness of extracts was evaluated through the comparison of the Reproduction Estimate of the groups "controlled and treated" for females, of mortality for the larvae, and of the calculation of the DL50 and the relative power for larvae and females. The extract of unripe fruits presented the effectiveness varying the $3.6 \%$ - $100 \%$ for females, and of ripe fruits it varied of $5.2 \%-99.7 \%$. The two extracts caused a mortality of $100 \%$ of the larvae in the highest concentrations. The extract of stored fruits presented effectiveness of $14 \%-83 \%$ on females, while the extract stored in refrigerator presented effectiveness of $8.4 \% 100 \%$ and both did not present larvicidal effect. The results demonstrate a superiority of the unripe extract, with a minor DL50 and superior power 1.497 times to the extract of ripe fruits.
\end{abstract}

KEY WORDS: Phytotherapy, ticks, control.

\section{RESUMO}

Extratos hexânicos obtidos dos frutos de Melia azedarach foram testados sobre fêmeas ingurgitadas e larvas de Rhipicephalus (Boophilus) microplus. Foram produzidos quatro extratos: um de frutos verdes e três com frutos maduros, sendo um com os frutos imediatamente processados, outro com frutos armazenados por cinco meses e um testado após armazenamento do extrato por 2 (dois) anos a $4^{\circ} \mathrm{C}$. Os testes foram feitos em triplicata, em diluições de 0,25\% a 0,0156\%. A eficácia dos extratos foi avaliada através da comparação da Reprodução Estimada dos lotes “controle e tratado" para fêmeas, da mortalidade para as larvas, e do cálculo das DL50 e

${ }^{1}$ Centro de Parasitologia Veterinária, Escola de Veterinária, Universidade Federal de Goiás (UFG), Campus II, Samambaia, Caixa Postal 131, Goiânia, GO 74001-970, Brasil. E-mail: lorenavet2@gmail.com

${ }^{2}$ Instituto de Química, UFG, Goiânia, GO, Brasil. da potência relativa para larvas e fêmeas. O extrato de frutos verdes apresentou uma eficácia variando de 3,6\% a 100\% para fêmeas, e o de frutos maduros variou de $5,2 \%$ a $99,7 \%$. Os dois extratos causaram uma mortalidade de $100 \%$ das larvas nas maiores concentrações. $\mathrm{O}$ extrato de frutos armazenados apresentou eficácia de $14 \%$ a $83 \%$ sobre fêmeas, enquanto o extrato armazenado em geladeira apresentou eficácia de 8,4\% a $100 \%$ e ambos não apresentaram efeito larvicida. Os resultados obtidos demonstram uma superioridade do extrato verde, com uma menor DL50 e potência 1,497 vezes superior ao extrato de frutos maduros.

PALAVRAS-CHAVE: Fitoterapia, carrapatos, controle.

\section{INTRODUÇÃO}

O carrapato Rhipicephalus (Boophilus) microplus (Canestrini, 1887) é um dos ectoparasitos mais importantes dos bovinos devido à sua ampla distribuição geográfica, ten- 
do se estabelecido dentro dos climas demarcados pelos paralelos $32^{\circ}$ Norte e Sul, com alguns focos nos paralelos $35^{\circ}$ norte e sul (KESSLER, 1998). As perdas econômicas devido a este parasito foram avaliadas, no Brasil, em US\$ 2 bilhões por ano (GRISI et al., 2002). Tais prejuízos são evidenciados, principalmente, pela espoliação sanguínea, que dependendo do tamanho da infestação, pode comprometer a produção de carne e leite e pela transmissão de agentes infecciosos, principalmente Anaplasma e Babesia spp, responsáveis pela tristeza parasitária bovina. O controle deste carrapato é realizado através da utilização de acaricidas com diferentes bases químicas, cujo uso constante e incorreto tem resultado em desenvolvimento de alelos resistentes nas populações de carrapatos, além de intoxicação dos animais e impacto ambiental pelo efeito residual deles na natureza (FURLONG, 1993).

Visando contornar o problema da resistência e tendo em vista a demanda dos consumidores por carne e leite sem resíduos químicos e a procura por produtos seguros para o meio ambiente, têm se observado um aumento significativo na procura por pesticidas botânicos. Entre os pesticidas fitoquímicos mais proeminentes estão aqueles oriundos da família Meliaceae. Melia azedarach, conhecida popularmente como cinamomo, é uma planta originária da Índia, bem adaptada às condições climáticas do Brasil e teve ação comprovada sobre diferentes pragas agrícolas e pecuárias (VALLADARES et al., 1999; JUAN et al., 2000, SOUZA; VENDRAMIM, 2001; BRUNHEROTTO; VENDRAMIM, 2001, BORGES et al., 2003).

Borges et al. (2003), avaliando a eficácia de extratos brutos de frutos maduros dessa planta, extraídos com diferentes solventes, sobre larvas e fêmeas de $R$. (B.) microplus, verificaram elevada taxa de mortalidade de larvas e alta eficácia sobre fêmeas ingurgitadas, sendo que o extrato não matou as fêmeas adultas, mas inibiu total ou parcialmente a produção de ovos e a embriogênese. Estes autores concluíram que extratos menos polares de frutos maduros de $M$. azedarach, utilizando hexano como solvente, à concentração de $0,250 \%$, apresentaram melhor eficácia tanto sobre fêmeas como sobre larvas deste carrapato. Em outro experimento, ao estudar a ação do extrato hexânico de frutos de $M$. azedarach sobre $R$. (B.) microplus em bezerros artificialmente infestados, Borges et al. (2005) observaram interferência da planta no desenvolvimento do carrapato sobre os animais infestados artificialmente. $\mathrm{O}$ fato de esta atividade ter sido verificada nos frutos torna interessante seu emprego como acaricida, principalmente no tocante da sustentabilidade ambiental, visto que sua utilização não implica no abate da planta, possibilitando um uso racional e a preservação das áreas de cultivo.

Diversos fatores podem influenciar a atuação de extratos naturais sobre controle de pragas. Alguns autores compararam a ação de extratos de frutos de $M$. azedarach, em diferentes estádios fisiológicos, sobre insetos de importância médica e agrícola, verificando que houve diferença de eficácia entre eles, sendo que em todos os trabalhos o extrato de frutos verdes foi superior ao de frutos maduros (VALLADARES et al.,
1.999; SOUZA; VENDRAMIM, 2001; BRUNHEROTTO; VENDRAMIM, 2001). Sabe-se também que existe uma forte influência biogeográfica na composição química de diversas plantas, inclusive para a M. azedarach. (ERMEL et al., 1986; MORGAN; THORNTON, 1973; ARIAS; HIRSCHMAN, 1988). Outro fator que influencia a bioatividade de extratos é o tempo de armazenamento da estrutura vegetal até o processamento (YAKKUNDI et al., 1995; JOHNSON et al., 1996).

Considerando a eficácia de extratos brutos de frutos de $M$. azedarach no controle do carrapato R.(B.) microplus, foi desenvolvido este experimento com o objetivo de comparar a ação extratos de frutos verdes e maduros desta planta, bem como a interferência do período de armazenamento dos frutos ou dos extratos em sua atividade acaricida.

\section{MATERIAIS E MÉTODOS}

Frutos verdes e maduros foram colhidos no Campus da Universidade Federal de Goiás (UFG), em Goiânia (16³4’24"S, 49¹7’32"W, 760 m), Goiás, Brasil. Uma exsicata desta planta está depositada no herbário da UFG, sob o número de registro 27.611. Os frutos após a secagem em estufa com circulação e renovação de ar, foram triturados em moinhos de facas rotativas e, em seguida, submetidos à extração por percolação a frio ou a quente, em Soxhlet, utilizandose como solvente o hexano. O solvente foi evaporado em bomba de vácuo. Foram produzidos dois extratos: um de frutos verdes (EFV) e um de frutos maduros (EFM). Parte dos frutos maduros foi armazenada por cinco meses em condições ambientes, com posterior processamento e produção de um terceiro extrato, denominado extrato de frutos maduros armazenados (EFMA). Os extratos foram testados sobre fêmeas ingurgitadas e larvas de $R$. (B.) microplus. Um quarto extrato, produzido anteriormente e armazenado em geladeira por dois anos, denominado extrato de frutos maduros armazenado em geladeira (EFMAG), também foi avaliado sobre instares deste carrapato.

Para a avaliação da eficácia dos extratos sobre as fêmeas ingurgitadas foram realizados testes de imersão preconizados por Drumond et al. (1973). Fêmeas ingurgitadas foram pesadas e separadas em grupos com pesos homogêneos de 10 teleóginas e imersas nas soluções previamente preparadas. Após cinco minutos, foram retiradas, secas e acondicionadas em placas de Petri para a oviposição. Após 20 dias, foi feita a coleta, pesagem e transferência dos ovos para uma seringa plástica, que aí permaneceram por mais 15 dias, para a avaliação da eclodibilidade. Após esse prazo foi feita a leitura visual de eclodibilidade dos ovos. A eficácia dos extratos foi determinada de acordo com o cálculo da reprodução estimada, proposto por Drumond et al. (1973).

Para a avaliação da eficácia dos extratos sobre as larvas foi utilizada a técnica do "sanduíche" preconizada por Shaw (1966). Aproximadamente 100 larvas de 7-21 dias de idade foram colocadas num circulo de papel filtro com $14 \mathrm{~cm}$ de 
diâmetro, dentro de uma placa de Petri. O extrato previamente diluído foi despejado ( $5 \mathrm{ml}$ ) sobre as larvas, que foram cobertas por outra folha de papel filtro e despejados mais $5 \mathrm{ml}$ do extrato. Após o tempo de imersão (10 minutos), os papéis foram retirados da placa, abertos e colocados em outra folha de papel filtro para secarem. Em seguida, as larvas foram transferidas para envelopes de papel filtro e mantidas na estufa até a leitura de sua viabilidade. A leitura da mortalidade das larvas ocorreu após 24, 72 e 168 horas da realização dos testes para o EFV, e após 168 horas para o EFM, EFMA e EFMAG.

Os extratos foram testados em triplicata, em cinco diluições ao dobro de $0,250 \%$ até $0,0156 \%$. Para cálculo das doses letais a 50\% (DL50) e das potências relativas foi utilizado o programa de análise de próbito Priprobit (Masayuki Sakuma, 1996-2000). Os resultados obtidos para o EFM foram utilizados como parâmetros de referência para cálculo das potências relativas.

\section{RESULTADOS E DISCUSSÃO}

Nos testes realizados para a avaliação dos extratos sobre fêmeas ingurgitadas foram observados os seguintes resultados: para o EFM os índices de produção de ovos e eclodibilidade variaram de $11 \mathrm{~g}$ a $47 \mathrm{~g}$ e de $0,7 \%$ a $91,7 \%$ com eficácia de 5,2\% a 99,7\%; para o EFV estes índices variaram de $2 \mathrm{~g}$ a $50 \mathrm{~g}$ e de $0 \%$ a $92,5 \%$, com eficácia variando de $3,6 \%$ a 100\% (Tabela 1). Já para o EFMA a produção de ovos e eclodibilidade variaram de 27 a 46 g e de 5 a 85\% com eficácias de 14 a 83\% (Tabela 1) enquanto para o EFMAG a vari- ação foi de 6,0g a 43,6g, 0,0 a 66,6\% respectivamente, com eficácia de $8,4 \%$ a $100,0 \%$.

Na avaliação dos extratos sobre larvas, tanto o EFV quanto o EFM promoveram a mortalidade de $100 \%$ das larvas testadas na mais alta concentração. O EFMA e o EFMAG não apresentaram efeito larvicida, sendo que os valores encontrados para mortalidade não diferiram dos encontrados nos grupos controles (Tabela 2). As DL50 para fêmeas ingurgitadas variam de $0,064 \%$ para o EFV a $0,123 \%$ para o EFMA e sobre larvas com leitura 168 hs após o teste de $0,003 \%$ para o EFV a 0,034\%, para o EFM. (Tabela 3). Houve um aumento da potência em relação ao EFM no EFV tanto para fêmeas (1,497 vezes) quanto para larvas (10,898 vezes) (Tabela 4).

Assim como observado no presente trabalho, a melhor eficácia dos frutos verdes também foi observada por Valladares et al. (1999) que estudando os efeitos da M. azedarach sobre Triatoma infestans (Hemiptera, Reduviidae) observaram que o extrato de frutos verdes apresentou forte efeito repelente, enquanto o extrato de frutos maduros não teve esta ação. Nenhum efeito sobre a postura de ovos, sobrevivência ninfal ou tempo de desenvolvimento foi observado para ambos os extratos. Souza e Vendramin (2001) avaliando a atividade inseticida de extratos aquosos de ramos, folhas, frutos verdes e frutos maduros de $M$. azedarach L. sobre ovos e ninfas da mosca-branca Bemisia tabaci (Hemiptera: Aleyrodidae) biótipo B, criada em tomateiro verificaram que os frutos verdes da planta foram a estrutura vegetal mais efetiva, seguindo-se as folhas e os frutos maduros. Ao estudar a bioatividade de extratos aquosos de $M$. azedarach sobre o desenvolvimen-

Tabela 1. Média e desvio padrão ( $\bar{x} \pm$ sd) da produção de ovos (PO), percentual de eclosão (PECL) e percentual de eficácia (PEF) dos EFM, EFV, EFMA e EFMAG sobre fêmeas ingurgitadas de $R$. (B.) microplus.

\begin{tabular}{|c|c|c|c|c|c|c|c|c|c|c|c|c|}
\hline \multirow[b]{2}{*}{$\begin{array}{c}\text { Conc } \\
(\%)\end{array}$} & \multicolumn{3}{|c|}{ EFM } & \multicolumn{3}{|c|}{ EFV } & \multicolumn{3}{|c|}{ EFMA } & \multicolumn{3}{|c|}{ EFMAG } \\
\hline & $\begin{array}{l}\text { PO } \\
(\%)\end{array}$ & $\begin{array}{c}\text { PECL } \\
(\%)\end{array}$ & $\begin{array}{l}\text { PEF } \\
(\%)\end{array}$ & $\begin{array}{l}\text { PO } \\
\text { (\%) }\end{array}$ & $\begin{array}{c}\text { PECL } \\
(\%)\end{array}$ & $\begin{array}{l}\text { PEF } \\
(\%)\end{array}$ & $\begin{array}{l}\mathrm{PO} \\
(\%)\end{array}$ & $\begin{array}{c}\text { PECL } \\
(\%)\end{array}$ & $\begin{array}{l}\text { PEF } \\
(\%)\end{array}$ & $\begin{array}{l}\text { PO } \\
\text { (\%) }\end{array}$ & $\begin{array}{c}\text { PECL } \\
(\%)\end{array}$ & $\begin{array}{l}\text { PEF } \\
(\%)\end{array}$ \\
\hline 0,25 & $11.2 \pm 7.3$ & $0.7 \pm 1.1$ & $99.7 \pm 0.4$ & $2.18 \pm 1.3$ & $0.0 \pm 0.0$ & $100 \pm 0.0$ & $26.7 \pm 3.0$ & $5.0 \pm 0.0$ & $83.3 \pm 14.3$ & $6.0 \pm 10.4$ & $0.0 \pm 0.0$ & $100 \pm 0.0$ \\
\hline 0,125 & $36.5 \pm 10.4$ & $46.7 \pm 18.9$ & $59.6 \pm 23.8$ & $25.6 \pm 6.6$ & $21.7 \pm 15.3$ & $88.9 \pm 7.3$ & $34.7 \pm 4.3$ & $50.0 \pm 5.0$ & $31.1 \pm 24.5$ & $31.2 \pm 12.7$ & $36.6 \pm 18.6$ & $54.8 \pm 22.7$ \\
\hline 0,0625 & $47.1 \pm 7.5$ & $85.0 \pm 10,0$ & $12.3 \pm 16.3$ & $39.6 \pm 5.9$ & $60.0 \pm 22.9$ & $46.7 \pm 24.0$ & $45.8 \pm 2.3$ & $80.0 \pm 10.0$ & $20.6 \pm 14.0$ & $41.6 \pm 8.3$ & $66.6 \pm 25.9$ & $8.4 \pm 27.5$ \\
\hline 0,0312 & $46.3 \pm 7.2$ & $91.7 \pm 5.8$ & $6.3 \pm 20.7$ & $50.7 \pm 2.4$ & $86.7 \pm 10.4$ & $3.7 \pm 3.8$ & $46.5 \pm 2.9$ & $85.0 \pm 10.0$ & $14.1 \pm 9.8$ & $43.6 \pm 14.2$ & $66.6 \pm 13.4$ & $9.2 \pm 17.9$ \\
\hline 0,0156 & $47.1 \pm 6.4$ & $90.0 \pm 5.0$ & $5.2 \pm 26.5$ & $46.1 \pm 4.2$ & $92.5 \pm 3.5$ & $3.6 \pm 4.8$ & & & & & & \\
\hline 0 & $47.7 \pm 4.5$ & $96.0 \pm 5.3$ & & $49.1 \pm 5.2$ & $92.7 \pm 4.6$ & & $50,3+2,2$ & $97,0+2,0$ & & $45,5+4,5$ & $86,6+6,4$ & \\
\hline
\end{tabular}

Tabela 2. Média e desvio padrão ( $\bar{x} \pm s d)$ da mortalidade de larvas de R.(B.) microplus tratadas com EFM e EFV de $M$. azedarach, 24, 72 e 168 horas após o tratamento.

\begin{tabular}{|c|c|c|c|c|}
\hline \multicolumn{5}{|c|}{$\%$ MORTALIDADE } \\
\hline \multirow{2}{*}{$\begin{array}{c}\text { Concentração } \\
(\%)\end{array}$} & \multicolumn{3}{|c|}{ EFV } & EFM \\
\hline & 24 & 72 & 168 & 168 \\
\hline 0.25 & $23.6 \pm 6.0$ & $50.3 \pm 2.6$ & $100 \pm 0.0$ & $100 \pm 0.0$ \\
\hline 0.125 & $52.4 \pm 4.8$ & $45.8 \pm 10.7$ & $100 \pm 0.0$ & $100 \pm 0.0$ \\
\hline 0.0625 & $13.3 \pm 1.0$ & $50.9 \pm 19.6$ & $100 \pm 0.0$ & $70.9 \pm 14.3$ \\
\hline 0.0312 & $19.6 \pm 4.1$ & $36.9 \pm 13.5$ & $91.5 \pm 5.5$ & $48.8 \pm 1.5$ \\
\hline 0.0156 & $5.6 \pm 5.0$ & $14.7 \pm 5.0$ & $95.6 \pm 3.8$ & $25.2 \pm 37.1$ \\
\hline 0.0 & $0.5 \pm 0.5$ & $1.1 \pm 1.1$ & $31.7 \pm 8.1$ & $5.2 \pm 1.1$ \\
\hline
\end{tabular}

OBS: não foram feitos testes com 24 e 72 horas com o EFM. to de Tuta absoluta (Lepidotpera: Gelechiidae), uma praga do tomateiro, Brunherotto e Vendramin (2001) observaram menor efeito causado pelo extrato de frutos maduros e sugeriram que, nesse estádio fisiológico, há menor quantidade de ingredientes ativos que nos frutos verdes, o que é coerente do ponto de vista de sobrevivência vegetal, uma vez que, nos frutos maduros, as sementes já estão completando a sua maturidade fisiológica e, por isso, têm menor necessidade de defesa química contra herbívoros.

Observa-se que o armazenamento dos frutos por cinco meses em condições ambientes causou um efeito deletério levando a um decréscimo de sua ação acaricida. Em um estudo realizado com Azadirachta indica, planta da mesma famí- 
Tabela 3. DL50 (\%) e intervalo fiducial 95\% dos extratos EFM, EFV e EFMA produzidos a partir do fruto de $M$. azedarach e testados sobre fêmeas e larvas de Boophilus microplus.

\begin{tabular}{ccccc}
\hline & \multicolumn{3}{c}{ DL50 } \\
\cline { 2 - 5 } & Fêmeas & \multicolumn{2}{c}{ Larvas } \\
\hline EFM & $0.0965(0.0644-0.1464)$ & & $24 \mathrm{hs}$ & $0.3804(0.1271-1.8347)$ \\
EFV & $0.0645(0.0430-0.0966)$ & EFV7 & 2 hs & $0.1182(0.0481-0.3205)$ \\
EFMA & $0.1236(0.0818-0,1904)$ & & $168 \mathrm{hs}$ & $0.0031(0.0001-0.0277)$ \\
EFMAG & $0.1059(0,0702-0,1614)$ & EFM & $168 \mathrm{hs}$ & $0.0343(0.0156-0.0590)$ \\
\hline
\end{tabular}

Tabela 4. Potência Relativa Estimada ao EFM e intervalo fiducial 95\% dos extratos EFV, EFMA e EFMAG produzidos a partir do fruto de $M$. azedarach e testados sobre fêmeas e larvas de Boophilus microplus.

\begin{tabular}{|c|c|c|c|}
\hline & \multicolumn{3}{|c|}{ Potência Relativa Estimada X EFM } \\
\hline & Fêmeas & & Larvas \\
\hline EFV & 1.497 (1.270-1.785) & 24 hs & $0.090(0.025-0.136)$ \\
\hline EFMA & $0.781(0.654-0.924)$ & EFV 72 hs & $0.0290(0.156-0.361)$ \\
\hline EFMAG & $0.911(0.769-1.083)$ & $168 \mathrm{hs}$ & $10.898(5.582-86.791)$ \\
\hline
\end{tabular}

lia da M. azedarach, Johnson et al. (1996) observaram um decréscimo na quantidade de azadirachtina e salanina, principais compostos identificados nos frutos, após um armazenamento de seis meses. Yakkundi et al. (1995), estudando somente a azadirachtina presente em sementes da mesma planta, constataram uma redução de 5\% na concentração do composto após um mês de armazenamento e de 35\% após quatro meses.

O armazenamento do extrato em geladeira também teve efeito deletério sobre a ação acaricida do mesmo. Observando as DL50 e potências relativas do EFMAG sobre fêmeas ingurgitadas e sobre larvas verifica-se que o armazenamento interferiu principalmente na ação larvicida. Desta forma é possível sugerir que as diferentes atividades, larvicida e sobre a produção de ovos e embriogênese, do extrato de frutos de $M$. azedarach sejam efetuadas por princípios ativos distintos. Um grande número de componentes bioativos já foi isolado de várias partes de plantas da família Meliacea. As azadirachtinas são os principais compostos químicos identificados nestas plantas já tendo sido relatadas em Azadirarchta indica e $M$. azedarach (BUTTERWORTH; MORGAN, 1968; MORGAN; THORNTON, 1973). Azadirachtinas e outros compostos bioativos podem exercer múltiplas ações sobre insetos, afetando alimentação, crescimento, desenvolvimento, reprodução entre outros fatores. A complexidade de componentes e múltiplos modos de ação por um lado representa um obstáculo para utilização da planta como inseticida, pois dificultam a identificação e síntese dos princípios ativos, mas por outro lado retarda o desenvolvimento de resistência (MULLA, 1999)

É importante ressaltar que os frutos empregados na produção dos extratos para este experimento foram obtidos das mesmas plantas utilizadas por BORGES et al. (2003) e atuaram sobre o carrapato $R$. (B.) microplus de forma semelhante, o que sugere que as plantas mantiveram as substâncias ativas estáveis. Vivan (2005) avaliando a eficácia de extratos hexânicos e aquosos de folhas e frutos de $M$. azedarach sobre o R. (B.) microplus, observou a melhor eficácia sobre fêmeas com extratos aquosos de frutos, na concentração de $0,10 \%$, atingindo 47,54\%. Com extratos hexânicos foi obtida a eficácia máxima de 31,89\%, na concentração de 0,10\%. Estes resultados são inferiores aos encontrados por Borges et al. (2003) e aos relatados neste trabalho. Esta diferença pode ocorrer devido a influências biogeográficas sofridas pelas plantas utilizadas. De acordo com Ermel (1986), A. indica apresenta grande variação individual no teor de azadiractina encontrada na semente. Este teor varia também dependendo da região de origem da planta. Exemplares de uma mesma espécie, colhidos em épocas diferentes, ou de locais diferentes, não têm necessariamente a mesma atividade biológica. Tais indícios foram observados para $M$. azedarach por alguns autores que identificaram diferentes compostos em frutos de plantas provenientes de diferentes regiões do mundo (MORGAN; THORNTON, 1973; ARIAS; HIRSCHMAN,1988; CABRAL et al., 1996). Ainda que orientada pelas características genéticas da planta, a síntese química das substâncias é controlada por fatores do ecossistema, iluminação, calor, constituição do solo, umidade (LAPA, 1999).

É interessante notar que apesar da relação filogenética próxima entre $M$. azedarach e $A$. indica, que pertencem à mesma família (Meliaceae), subfamília (Melioideae) e tribo (Meliae), óleos essenciais $A$. indica não apresentaram atividade sobre larvas de $R$. (B.) microplus nem mesmo a soluções a 10\% (CHAGAS et al., 2007). Diferentemente, Borges et al. (2003) obtiveram, com extratos de $M$. azedarach, 100\% de mortalidade sobre larvas deste carrapato, assim como foi observado no presente estudo.

Diante dos resultados obtidos conclui-se que os frutos verdes de $M$. azedarach são a melhor alternativa para a utilização da planta no controle do $R$. (B.) microplus. O armazenamento interfere negativamente na atividade dos extratos e se necessário deve ser feito após o processamento dos frutos. Visando a estabilização dos princípios ativos novos estudos serão feitos com concentrados emulsionáveis do extrato de frutos verdes para testes em animais infestados com R. (B.) microplus.

\section{REFERÊNCIAS BIBLIOGRÁFICAS}

ARIAS, A.R.; HIRSHMANN, C.S. The effects of Melia azedarach on Triatoma infestans bugs. Fitoterapia, v.59, n. 2, p. 148-149, 1988.

BORGES, L.M.F.; FERRI, P.H.; SILVA, W.J.; SILVA, W.C.; SILVA, J.G. In vitro efficacy of extracts of Melia azedarach against the tick Boophilus microplus. Medical and Veterinary Entomology, v. 17, n. 2, p. 228-231, 2003.

BORGES, L.M.F.; FERRI, P.H.; SILVA, W.C.; SILVA, W.J. Ação do Extrato Hexânico de Frutos Maduros de Melia azedarach (Meliaceae) sobre Boophilus microplus (Acari: Ixodidae) em bezerros infestados artificialmente. Revista de Patologia Tropical, v. 34, n. 1, p.53-59, 2005. 
BRUNHEROTTO, R.; VENDRAMIM, J.D. Biotividade de Extrato Aquosos de Melia azedarach L. sobre o desenvolvimento de Tuta absoluta (Meyrick) (Lepidoptera: Gelechiidae) em tomateiro. Neotropical Entomology, v. 30, n.3, p. 455-459, 2001.

BUTTERWORTH, J.H.; MORGAN, E.D. Isolation of substances that suppress feedings in locusts. Chemical Comunications, v. 28, n. 1, p.23-24, 1968.

CABRAL, M.M.O.; HEINZ REMBOLD, E.S.G.; SIMONE, S.G.D.; KALECOM, A. Anti-moulting activity in Brazilian Melia azedarach. Memórias do Instituto Oswaldo Cruz, Rio de Janeiro, v. 91, n. 1, p. 117-118, 1996.

CHAGAS, A.C.S.; OLIVEIRA, M.C.S.; FORIM, M.R.; SILVA, M.F.G.F.; GIGLIOTI, R.; MIGLIATO, M.A.T. Avaliação do óleo de neem com teor padronizado de azadirachtina-a sobre as larvas de Rhipicephalus (Boophilus) microplus. In: CONGRESSO BRASILEIRO DE VETERINÁRIA, 34, 2007, Santos. Anais...Santos: CONBRAVET, 2007 CD ROM.

DRUMMOND, R.O.; ERNST, S.E.; TREVINO, J.L.; GLADENY, W.J.; GRAHAM, O.H. Boophilus annulatus and Boophilus microplus: laboratory tests for inseceticides. Journal of Economic Entomology, v. 66, n. 1, p. 130- 133, 1973.

ERMEL, K.; PAHLICH, E.; SCHMUTTERER, H. Azadirachtin content of neem kernels from different geographical locations, its dependence on temparature, relative humidity and light. In: INTERNATIONAL NEEM CONFERENCE, 3, 1986, Eschborn. Proceedings... Eschborn:GTZ, 1986. p.171-184.

FURLONG, J. Controle do carrapato dos bovinos na Região Sudeste do Brasil. Caderno Técnico da Escola de Veterinária da UFMG, v. 8, p.49-61, 1993.

GRISI, L.; MASSARD, C.L.; BORJA, G.E.M.; PEREIRA, J.B. Impacto econômico das principais ectoparasitoses em bovinos no Brasil. Hora Veterinária, v. 21, n. 125, p. 8-10, 2002.

JOHNSON, S.; MORGAN, E.D.; PEIRIS, C.N. Development of the Major Triterpenoids and Oil in the Fruit and Seeds of Neem (Azadirachta indica). Annals of Botany, v. 78, n. 3, 383-388, 1996.

JUAN, A.; SANS, A.; RIBA, M. Antifeedant Activity of Fruit and Seed Extracts of Meliaazedarach and Azadirachta indica on Larvae of Sesamia nonagrioides. Phytoparasitica, v. 28, n. 4, p. 1-9, 2000.

KESSLER, R.H.; SCHENK, M.A.M. Carrapato, tristeza parasitária e tripanossomose dos bovinos. Campo Grande: Embrapa-CNPGC, 1998. 157 p.

LAPA, A.J.; SOUCCAR, C.; LIMA-LANDMAN, M.T.R.; GODINHO, R.O.; LIMA T.C.M. Farmacologia e toxicologia de produtos naturais. In: SIMÕES, C.M.O., SCHENKEL, E.P.; GOSMAN, G.; MELLO, J.C.P.; MENTZ, L.A.; PETROVICK, P.R. Farmacognosia: da planta ao medicamento. 1.ed. Porto Alegre: UFRGS, 1999. p. 181-196.

MULLA, M.S.; SU, T. Activity and biological effects of neem products against arthropod of medical and veterinary importance. Journal of American Mosquito Control Association, v. 15, n. 1, p. 133-152, 1999.

MORGAN, E.D.; THORNTON, M.D. Azadirachtin in the fruit of Melia azedarach. Phytochemistry, v. 12, n. 1, p. 391392, 1973.

SHAW, R.D. Culture of an organophosphorus resistant strain of Boophilus microplus (Can.). Bulletin Entomological Research, v. 56, n. 3, p. 389-404, 1966.

SOUZA, A.P.; VENDRAMIM, J.D. Atividade inseticida de extratos aquosos de meliáceas sobre a mosca-branca Bemisia tabaci (Genn.) biótipo B (Hemiptera: Aleyrodidae). Neotropical Entomology, v. 30, n. 1, 133137, 2001.

VALLADARES, G.R.; FERREYRA, D.; DEFAGO, M.T.; CARPINELLA, M.C.; PALACIOS, S. Effects of Melia azedarach on Triatoma infestans. Fitoterapia, v.70, n. 4, p.421-424, 1999.

YAKKUNDI, S.R.;THEJAVATHI, R.; RAVINDRANATH, B. Variation of Azadirachtin Content during Growth and Storage of Neem (Azadirachta indica) Seeds. Journal of Agricultural Food and Chemistry, v. 43, n. 9, p. 2517-2519, 1995.

VIVAN, M.P. Uso do cinamomo (Melia azedarach) como alternativa aos agroquímicos no controle do carrapato bovino (Boophilus microplus). 2005. 72f. Dissertação (Mestrado) - Universidade Federal de Santa Catarina, Florianópolis, 2005.

Recebido em 13 de agosto de 2007.

Aceito para publicação em 21 de março de 2008. 